\title{
Identificação precoce da sepse pela equipe de enfermagem em Unidades de Terapia Intensiva através dos sinais e sintomas: revisão narrativa
}

\author{
Early identification of sepsis by the nursing team in Intensive Care Units through signs and \\ symptoms: narrative review
}

Identificación precoz de sepsis por el equipo de enfermería en Unidades de Cuidados Intensivos a través de signos y síntomas: revisión narrativa

\section{Resumo}

A Sepse é definida como uma disfunção do organismo causada por uma resposta desregulada na infecção, em diferentes estágios clínicos de um mesmo processo fisiopatológico. O presente estudo teve como objetivo descrever quais os sinais e sintomas que antecedem a sepse os profissionais de enfermagem podem identificar de forma precoce em pacientes internados em Unidades de Terapia Intensiva - UTI's. Metodologia: Trata-se de uma revisão narrativa da literatura fundamentada a partir de uma pesquisa bibliográfica a partir de uma pesquisa bibliográfica realizada em 
julho de 2020 nas respectivas fontes de dados: Medical Literature Analysis and Retrieval System Oline (Medline) e Scientific Electronic Library Online (SciELO). Resultados: O enfermeiropor trabalhar diretamente com o cuidado ao paciente beira-leito, tem a possibilidade de identificar as primeiras alterações clínicas dos sinais e sintomas que antecedem a sepse como por exemplo: taquicardia (88,5\%), taquipnéia $(79,2 \%)$, oligúria $(78,1 \%)$, hipotermia $(75,2 \%)$, hipotensão $(67,1 \%)$, hipertermia $(30,2 \%)$, dessaturação $(12,7 \%)$, Rebaixamento do Nível de Consciência (RNC) $(5,5 \%)$. Contudo, os profissionais de enfermagem tem certa dificuldade na identificação precoce da sepse na Unidade de Terapia Intensiva (UTI), seja pela complexidade que o paciente se encontra, falta de protocolo em algumas instituições ou até mesmo pela falta de conhecimento sobre este processo clínico.

Palavras-chave: Enfermagem; Sepse; Sinais e sintomas; Terapia intensiva.

\begin{abstract}
Sepsis is defined as a dysfunction of the organism caused by a deregulated response to infection, in different clinical stages of the same pathophysiological process. The present study aimed to describe the signs and symptoms that precede sepsis that nursing professionals can identify early in patients hospitalized in Intensive Care Units - ICUs. Methodology: This is a narrative review of the literature based on a literature search based on a literature search carried out in July 2020 in the respective data sources: Medical Literature Analysis and Retrieval System Oline (Medline) and Scientific Electronic Library Online (SciELO). Results: Because nurses work directly with bedside patient care, they are able to identify the first clinical changes in signs and symptoms that precede sepsis, such as: tachycardia $(88.5 \%)$, tachypnea $(79.2 \%)$, oliguria $(78.1 \%)$, hypothermia $(75.2 \%)$, hypotension $(67.1 \%)$, hyperthermia (30.2\%), desaturation (12.7\%), Lowering of the Level of Consciousness (RNC) (5.5\%). However, nursing professionals have some difficulty in the early identification of sepsis in the Intensive Care Unit (ICU), either because of the complexity of the patient, lack of protocol in some institutions or even lack of knowledge about this clinical process.
\end{abstract}

Keywords: Nursing; Sepsis; Signs and symptoms; Intensive therapy.

\title{
Resumen
}

La sepsis se define como una disfunción del organismo provocada por una respuesta desregulada a la infección, en diferentes estadios clínicos de un mismo proceso fisiopatológico. El presente estudio tuvo como objetivo describir los signos y síntomas que preceden a la sepsis que los profesionales de enfermería pueden identificar precozmente en pacientes internados en Unidades de Cuidados Intensivos - UCI. Metodología: Se trata de una revisión narrativa de la literatura basada en una búsqueda bibliográfica realizada en julio de 2020 en las respectivas fuentes de datos: Medical Literature Analysis and Retrieval System Oline (Medline) y Scientific Electronic Library Online (SciELO). Resultados: Debido a que las enfermeras trabajan directamente con el cuidado del paciente al pie de la cama, pueden identificar los primeros cambios clínicos en los signos y síntomas que preceden a la sepsis, como: taquicardia $(88,5 \%)$, taquipnea $(79,2 \%)$, oliguria $(78,1 \%)$, hipotermia $(75,2 \%)$, hipotensión $(67,1 \%)$, hipertermia $(30,2 \%)$, desaturación (12,7\%), Disminución del Nivel de Conciencia (RNC) (5,5\%). Sin embargo, los profesionales de enfermería tienen alguna dificultad en la identificación temprana de sepsis en la Unidad de Cuidados Intensivos (UCI), ya sea por la complejidad del paciente, falta de protocolo en algunas instituciones o incluso falta de conocimiento sobre este proceso clínico.

Palabras clave: Enfermería; Septicemia; Signos y síntomas; Terapia intensiva.

\section{Introdução}

A Sepse é definida como uma disfunção do organismo causada por uma resposta desregulada na infecção. Pode se manifestar em diferentes estágios clínicos de um mesmo processo fisiopatológico. Esse processo é considerado como um desafio enfrentado constantemente peloso profissionais de enfermagem devido à complexidade no desfecho clínico para um reconhecimento e tratamento precoce (Lelis et al., 2017).

Analisando o cenário das Unidades de Terapia Intensiva (UTI’s), a sepse, o choque séptico e o disfuncionamento de múltiplos órgãos são os maiores motivos de morte entre todas as doenças que acometem pacientes graves e críticos. Atualmente, cerca de $25 \%$ dos leitos estão tomados por pacientes em quadros sépticos no Brasil (Ribeiro, Gonçalves \& Pereira, 2018).

As UTI's são consideradas como um setor de alta complexidade que assisti os pacientes com prognósticos críticos que necessitam de cuidados invasivos. Nesse setor, encontramos os maiores aparatos tecnológicos da instituição hospitalar e essa 
assistência deve dispor de recursos materiais e humanos especializados para a demanda em estado grave, como por exemplo, sepse (Santos et al., 2016; Freitas, et al., 2017).

$\mathrm{Na}$ incidência da sepse, estudos mostram que há um aumento desproporcionalmente em adultos mais velhos e mais do que 50\% dos casos de sepse ocorre em adultos com mais de 65 anos de idade. O sexo feminino parece ter um menor risco de desenvolvimento da sepse do que em homens. Os pacientes que sobrevivem o quadro clínico de sepse estão mais propensos a serem internados para cuidados intensivos e/ou centros para cuidados prolongados no primeiro ano após a primeira internação e também parecem ter uma diminuição persistente na qualidade de vida (Rosa, et al. 2018).

Dados fornecido pelo Instituto Latino Americanos da Sepse (ILAS) evidencia que a taxa de mortalidade relacionada à sepse, nas instituições hospitalares públicas e privadas brasileiras, tem uma variância de 30\% a 70\%. Neste ambiente de cuidados intensivos, tem um risco aumentado para o desenvolvimento da sepse, devido aos vários fatores relevantes para o seu desencadeamento, um dos fatores relacionados a mortalidade de paciente com sepse, incluem o tempo até o início dos antibióticos, o controle da infecção e a infusão de fluidos e fatores intrínsecos ao paciente, como a idade e as comorbidades (Freitas, et al. 2017).

Cabe ao profissional de enfermagem a identificação precoce dos sinais e sintomas, para planejar, coordenar e implementar ações diante das diferentes situações clínicas relativos à sepse não só pelo diagnóstico, mas também para colaborar de forma rápida com os planos terapêuticos e estratégias de monitorização, melhorandoo prognóstico do pacientes (Goulart, et al. 2019; Silva et al., 2018).

Nesse contexto, entende-se que a identificação precoce e adequada torna-se crucial para o sucesso na abordagem do paciente séptico, diminuindo assim a incidência de disfunções orgânicas por meio da assistência sistemática da enfermagem na busca contínua pela detecção precoce de pacientes hospitalizados na fase inicial da síndrome. Nesta perspectiva o enfermeiro da Unidade de Terapia Intensiva que estar diretamente envolvida (o) neste processo clínico da sepse, em seu atendimento deve ser capaz de reconhecer os sinais e sintomas de gravidade, e relatar as alterações presentes de forma imediata para que o tratamento possa ser iniciado (Neto, et al. 2019; Souza et al., 2020).

$\mathrm{Na}$ tentativa de contribuir para a sensibilização das (os) enfermeiras (os) na importância da identificação dos sinais e sintomas da sepse, que favoreça a redução da sua mortalidade na Unidade de Terapia Intensiva, este estudo estruturou-se com objetivo de descrever quais os sinais e sintomas que antecedem a sepse os profissionais de enfermagem podem identificar de forma precoce em pacientes internados em UTI's.

\section{Metodologia}

O presente estudo trata-se de uma revisão narrativa da literatura. Essa pesquisa foi fundamentada a partir de pesquisa bibliográfica nas respectivas fontes de dados: Medical Literature Analysis and Retrieval System Oline (Medline) e Scientific Electronic Library Online (SciELO) e Google acadêmico.

Tendo em vista a importância da equipe de enfermagem no diagnóstico precoce da sepse, foi elaborada como questão norteadora para a presente revisão narrativa a seguinte questão: "Quais os sinais e sintomas que antecedem a sepse os profissionais de enfermagem podem identificar de forma precoce em pacientes internados em UTI's?". A busca na base de dados foi realizada no período do mês de julho de 2020, utilizando os Descritores em Ciência de Saúde (DeCS) que melhor se encaixavam nos objetivos propostos. Sendo assim, os descritores selecionados em língua portuguesa foram "Enfermagem"; "Sepse"; "Sinais e sintomas" e "Terapia intensiva", sendo utilizado o operador booleano AND para realização da chave de busca. 
Os critérios de inclusão estabelecidos para a seleção foram publicações disponíveis na íntegra de forma gratuita, no idioma português, publicados no período de 2016 a 2020 e que abordassem o assunto equivalente ao da pesquisa. Como os critérios de exclusão utilizaram-se publicações indisponíveis na íntegra de forma gratuita, e aquelas que não abordavam o objetivo desejado nesta pesquisa.

Foram encontrados trinta e cinco artigos nas fontes de dados: sete referentes à Medline; um da SciELO e vinte e sete do Google acadêmico. A seleção dos estudos utilizados foi baseada respectivamente na leitura do seu título, resumo, e o texto completo, que ao final foram selecionadas vinte e uma produções científicas, visto que, atenderam aos critérios de inclusão. Posteriormente, mediante o reconhecimento, seleção e ordenação das informações dos documentos realizou-se o processo de leitura do material viabilizando o entendimento e a compreensão em relação à temática proposta na pesquisa.

\section{Resultados e Discussão}

A sepse é considerada uma doença fatal e um problema de saúde pública, levando a uma estimativa de 600 mil novos casos de sepse a cada ano no Brasil e 16,5\% dos atestados de óbitos emitidos, girando em torno de 250 mil casos de mortalidade (Silva \&, Souza, 2018).Neste contexto, existem barreiras que dificultam a identificação, controle e prevenção, sendo que nos estudos analisados para a construção da pesquisa é enfatizada a necessidade da criação/implantação de protocolos de resposta rápida, que tem o objetivo de auxiliar a assistência de enfermagem diante do paciente com alterações sugestivas de sepse, permitindo uma abordagem precoce e segura para intervir no processo de agravamento do estado clínico do paciente (Oliveira, et al., 2019; Morello, et al., 2019; Alvim, et al., 2020).

É de extrema importância que o enfermeiro conheça sobre a fisiopatologia da doença e sua evolução para poder reconhecer e atender de forma ágil e segura o paciente com sepse, prevenindo assim sequelas, aumentando a sobrevida e agindo no processo das repercussões clínicas. Para o reconhecimento precoce dos sinais e sintomas, faz-se necessário a aplicação da Sistematização da Assistência de Enfermagem (SAE) e utilização dos protocolos constituídos nas instituições hospitalares, tornando-se esses dois métodos imprescindíveis para uma assistência de qualidade (Ribeiro, Gonçalves \&, Pereira, 2018; Lima et al., 2016).

De acordo com Fernandes et al. (2018) em fevereiro de 2016 a Society of Critical Care Medicine (SCCM) e a European Society of Critica lCare Medicine (ESICM) publicaram uma nova definição de sepse, anteriormente conhecida como Síndrome de Inflamação Respiratória Sistêmica (SIRS), atualmente sendo definida como a avaliação da disfunção orgânica, analisado um aumento de dois itens no escore Sequential Organ Failure Assessment (SOFA), que avalia disfunção de 6 sistemas do corpo através de exames laboratoriais. A partir disto, diverge que pacientes somente com hipotensão ou com escala de coma de Glasgow abaixo de 13 não seriam considerados como sepse (Morello, et al., 2019; Westphal, et al., 2018).

Entretanto, teve uma modificação no SOFA, o quick SOFA (qSOFA), que avalia 3 critérios de forma precoce em paciente graves com suspeita de sepse beira-leito. O profissional de enfermagem deve considerar o resultado SOFA positivo quando evidenciar no paciente a FR > 22/incursões respiratórias por minuto, nível de consciência inferior a 15 na escala de Glasgow ou quando a pressão arterial sistólica for $<$ de $100 \mathrm{mmHg}$. Nesse caso a sepse deve ser considerada grave (Garrido, et al., 2017).

Os critérios a serem avaliados na suspeita de sepse são: a hipotensão, pressão arterial sistólica $<90 \mathrm{mmHg}$ ou PAM $<$ 65 mmHg ou queda de PA > 40 mmHg, oligúria ou elevação da creatinina; relação PaO2/FiO2 < 300 ou a necessidade de O2 para manter SpO2 > 90\%; contagem de plaquetas $<100.000 / \mathrm{mm}^{3}$ ou redução de 50\% no número de plaquetas em relação ao maior valor registrado nos últimos 3 dias; acidose metabólica: déficit de bases $\leq 5,0 \mathrm{mEq} / \mathrm{L}$ e lactato acima do valor de 
referência; alteração do nível de consciência; aumento significativo de bilirrubinas (Rosa, et al., 2018; Oliveira, et al., 2019; Goilart, et al., 2019).

Neste contexto o papel do enfermeiro é primordial, já que estes profissionais estão presentes à beira leito do paciente. O enfermeiro é o profissional que está mais próximo do paciente dentro das 24 horas nas Unidades de Terapia Intensiva e que tem como vantagem a observação e o acompanhamento da sua evolução clínica de perto. Os demais profissionais (Fisioterapeutas, Médicos) embora realizem procedimentos a beira leito não tem essa vantagem, pois realizam visitas esporádicas durante a sua jornada de trabalho (Sá, et al., 2019; Silva, et al., 2017). Entretanto, devido a sua proximidade com o paciente para realização da assistência, pode ser o profissional primário a identificar as manifestações clínicas da sepse.

No estudo de Moura et al. (2017), foram utilizados 347 prontuários. Os pacientes que desenvolveram sepse após a internação nas UTIs apresentaram diversos tipos de sinais e sintomascomo taquicardia (88,5\%), taquipneia (79,2\%), oligúria $(78,1 \%)$, hipotermia $(75,2 \%)$, hipotensão $(67,1 \%)$, leucocitose $(48,4 \%)$, hipertermia $(30,2 \%)$, dissaturação $(12,7 \%)$, Rebaixamento do Nível de Consciência (RNC) $(5,5 \%)$ e leucopenia $(4,9 \%)$ sendo que alguns pacientes foram acometidos por mais de um sintoma.

A pesquisa de (Lima et al., 2016) corrobora com o estudo supracitado e trás novos sinais e sintomas que antecede a sepse que os profissionais de enfermagem podem está se deparando no cuidado ao paciente critico, classificando-as como sinais e sintomas gerais: hipertermia ou hipotermia, edemas, hiperglicemia, dificuldade ao respirar; já as inflamatórias são a leucocitose, aumento da proteína $\mathrm{C}$ ativa ou leucopenia; e as hemodinâmicas como hipotensão arterial baixa, débito cardíaco (DC) aumentado e saturação venosa(SvO2) baixa.

Diante das alterações citadas acima, os profissionais de enfermagem devem está atento a qualquer mudança na clínica do paciente, pois esses parâmetros clínicos podem indicar sepse. Todavia, o seu reconhecimento muitas vezes ainda não é feito em um tempo oportuno, deixando o organismo do paciente mais propenso a ocorrência de disfunção de múltiplos órgão e sistemas. Visto isso, a identificação dos sinais e sintomas precocemente com ações e intervenções rápidas influencia diretamente nos índices de morbimortalidade à sepse (Silva et al., 2018)

A sepse exige, assim, uma visão holística e integral na assistência dos profissionais de enfermagem. No decorrer do estudo de Lima, et al. (2020), realizado em um hospital escola entre julho a agosto de 2017, foram entrevistados 47 enfermeiros da UTI, identificando-se que os enfermeiros de uma unidade de cuidados intensivos têm conhecimentos sobre a prevenção e o controle da sepse, contudo, demonstraram alguns défices. A falta de conhecimento sobre o quadro clínico pode atrasar o diagnóstico, conduzindo a um agravamento do estado geral do paciente (Freitas, et al. 2017).

Nessa perspectiva, a (o) enfermeira (o), por trabalhar diretamente com o cuidado ao paciente, beira-leito, tem a possibilidade de identificar as primeiras alterações clínicas dos sinais e sintomas que antecedem a sepse. O Instituto Latino Americano da Sepse (ILAS) recomenda que diante da sepse o acionamento da equipe médica é a conduta que deve ser adotada para dar continuidade na assistência ao paciente séptico. O profissional de enfermagem deve estar capacitado para distinguir os sinais e sintomas de forma a possibilitar que o profissional médico seja acionado (Branco, et al., 2020; Souza, et al., 2020).

No entanto, a patologia em estudo continua sendo cada vez mais um problema de saúde, fazendo-se emergir na necessidade de termos equipes formadas, capacitadas e que atuem efetivamente. Para tanto, se faz necessário uma constante procura no conhecimento em atualizações, pois o investimento na formação é fundamental para prevenir, identificar e combater a sepse (Alvim, et al., 2020; Goulart, et al., 2019). 


\section{Conclusão}

A sepse tem uma prevalência hospitalar crescente e representa atualmente um verdadeiro problema de saúde publica. Através desta pesquisa, percebeu-se que os profissionais de enfermagem tem certa dificuldade na identificação precoce da sepse em UTI's, seja pela complexidade que o paciente séptico se encontra, falta de protocolo em algumas instituições hospitalares ou até mesmo pela falta de conhecimento sobre este processo clínico. Entretanto, a educação permanente é indispensável para o conhecimento deste desfecho clínico, pois, o enfermeiro tem total responsabilidade na notificação dos quadros de sepse e na assistência inicial juntamente com a equipe multidisciplinar.

A partir daí, julga-se necessária e indispensável à atenção voltada para os sinais e sintomas e não só os exames laboratoriais a fim de ter uma identificação precoce dos pacientes com quadro de sepse, visto que, a mesma traz consequências desastrosas para o paciente e interfere diretamente em sua recuperação, pois foi evidenciada uma taxa de 16,5\% de óbito pela patologia em estudo.

Nesse contexto, vale fomentar que as intervenções de enfermagem devem ser orientadas de acordo com as alterações dos parâmetros clínicos cardiorrespiratórios e sinais e sintomas que são clássicos da sepse para promover um melhor prognostico clínico dos pacientes submetidos a este quadro.

\section{Referências}

Alvim, A. L. S., Silvano, L. M., Ribas, R. T. M., \& Rocha, R. L. P. (2020). Conhecimento da Equipe de Enfermagem em Relação aos Sinais e Sintomas da Sepse. Enferm. Foco, 11(2), 133-138.

Branco, M. J. C. , Lucas, A. P. M., Marques, R. M. D., \& Sousa, P. P. (2020). O Papel do Enfermeiro Perante o Paciente Crítico com Sepse. Rev Bras Enferm, 73(4), 1-8.

Fernandes, A. M. G., Soares, G. T. M., Nascimento, L.K. A. S., Pellense, M. C. S., Carvalho, G. A. F. L., \& Sena, D. C. S. (2017/2018). Atuação da Enfermagem na Detecção Precoce e Tratamento da Sepse na Terapia Intensiva. Revista Humano Ser-UNIFACEX, 1(1), 66-83.

Freitas, A. P. O., Nunes, I. T. O., Santana, S. M. S., Ferrari, Y. A. C., \& Cavalcante, A. B. (2017). Importância do Papel do Enfermeiro Frente a Sepse. Universidade Tiradentes.

Garrido, F., Tieppo, L., Pereira, M. D. S., Freitas, R., Freitas, W. M., Filipini, R., Coelho, P. G., Fonseca, L. A. F. \& Fiorano, A. M. M. (2017). Ações do Enfermeiro na Identificação Precoce de Alterações Sistêmicas Causadas pela Sepse Grave. ABCS Health Sci, 42(1), 15-20.

Goulart, L. S., Júnior, M. A. F., Sarti, E. C. F. B., Sousa, A. F. L. S., Ferreira, A. M., \& Frota, O. P. (2019). Os Enfermeiros Estão Atualizados para o Manejo Adequado do Paciente com Sepse? Esc. Anna Nery, 23(4).

Lelis, L. S., Amaral, M. S., \& Oliveira, F. M. (2017) As Ações de Enfermagem Frente à Sepse, uma Abordagem do Paciente Crítico: Uma Revisão da Literatura. Revista Científica FacMais, 11(4), 50-66.

Lima, A. C. S. L., \& Picanço, C. M. (2016). Intervenções de Enfermagem no Controle da Sepse na Unidade de Terapia Intensiva. Trabalho de Conclusão de curso - Faculdade Estácio, Bahia.

Lima, J. C. C., Filho, L. M. M., Santos, T. N., Silva, C. S., Melchior, L. M. R., \& Sousa, T. V. (2020). Sepse e Choque Séptico: Compreensão de Enfermeiros de um Hospital Escola de Grande Porte. Revisa, 9(2), 254-61.

Morello, L. G., Costa-Dalla, L. M., Fontana, R. M., Netto, A. C. S. O., Petterle, R. R., Conte, D., Pereira, L. A., Krieger, M. A., \& Raboni, S. M. (2019). Avaliação das Características Clínicas e Epidemiológicas de Pacientes com e Sem Sepse nas Unidades de Terapia Intensiva de um Hospital Terciário. einstein (São Paulo), 17(2), 1-8.

Moura, J. M., Bertolli, E. S., Pereira, R. M., Frutuoso, I. S., Werneck, A. L., Contrin, L. M. (2017). Diagnóstico de Sepse em Pacientes após Internação em Unidade de Terapia Intensiva. Arq. Ciênc. Saúde, 24(3), 55-60.

Neto, J. M. R., Almeida, A. R. M., Silva, L. M., \& Viana, R. A. P. P., Nóbrega, M. M. (2019). Paciente Grave com Sepse: Concepções e Atitudes de Enfermeiros Intensivistas. Enferm Bras, 18(5), 650-657.

Oliveira, S. C., Corrêa, B. T., Dodde, H. N., Pereira, G. L., \& Aguiar, B. G. C. (2019). O Enfermeiro na Detecção dos Sinais e Sintomas que Antecedem Sepse em Pacientes na Enfermaria. J. res.: fundam. care. Online, 11(5).

Ribeiro, J. A. R., Gonçalves, M. S., \& Pereira, G. C. S. (2018). Ações do Enfermeiro na Identificação Precoce da Sepse. Enfermagem Revista, 21(2):27-40.

Rosa, R. S., Silva, O. C., Picanço, C. M., Biondo, C. S., Andrade, D. M. B., \& Prado, I. F. (2018). Intervenções de Enfermagem nas Alterações dos Parâmetros Clínicos Cardiorrespiratórios em Pacientes com Sepse. Rev Enferm UFSM, 8(2), 399-409. 
Research, Society and Development, v. 11, n. 3, e19111325855, 2022

(CC BY 4.0) | ISSN 2525-3409 | DOI: http://dx.doi.org/10.33448/rsd-v11i3.25855

Sá, L. T. G. S., Souza, A. M., Santos, J. P. A., Oliveira, J. C. (2019). Análise da Assistência de Enfermagem nos Cuidados aos Pacientes com Sepse na Unidade de Terapia Intensiva. Universidade Tiradentes.

Santos, A. M., Souza, G. R. B., \& Oliveira, A. M. L. (2016). Sepse em Adultos na Unidade de Terapia Intensiva: Características Clínicas. Arq Med Hosp Fac Cienc Med Santa Casa São Paul, 61, 3-7.

Silva, A. P. R. M., \& Souza, H. V. (2018). Sepse: Importância da Identificação Precoce pela Enfermagem. Revista Pró-univerSUS, 09(1), 97-100.

Silva, T. S. C., Rodrigues, J. L. N., Amaral, G. P., \& Júnior, A. A. P. (2017). Conhecimento dos Profissionais de Enfermagem sobre Sepse - Estudo em um Hospital Universitário de Fortaleza/Ceará. Rev Med UFC, 57(3), 24-29.

Souza, A. P. C., Garcia, R. A. S., Neto, M. F. S. (2020). Assistência de Enfermagem em Unidade de Terapia Intensiva nas Alterações Sistêmicas Causadas pela Sepse. Braz. J. Hea. Ver., 3(5), 11398-11404.

Westphal, G. A., Pereira, A. B., Fachin, S. M., Sperotto, G. Gonçalves, M., Albino, L., Bittencourt, Rodolfo, Franzini, V. R., \& Koenig, A. (2018). Um Sistema Eletrônico de Alerta Ajuda a Reduzir o Tempo para Diagnóstico de Sepse. Rev Bras Ter Intensiva,30(4), 414-422. 